\title{
Autologous stem cell therapy in knee osteoarthritis: a systematic review of randomised controlled trials
}

\author{
Tom GH Wiggers (1) ,' Marinus Winters 다, ${ }^{2}$ Noortje AC Van den Boom 다, ${ }^{3}$ \\ Hidde J Haisma, ${ }^{4}$ Maarten $\mathrm{H}$ Moen ${ }^{(1)}{ }^{5}$
}

- Additional online supplemental material is published online only. To view please visit the journal online (http://dx.doi.org/10.1136/ bjsports-2020-103671).

${ }^{1}$ Sports Medicine, St Anna Hospital, Geldrop, The Netherlands

${ }^{2}$ Research Unit for General Practice in Aalborg, Department of Clinical Medicine, Aalborg Universitet Det Sundhedsvidenskabelige Fakultet, Aalborg, Denmark ${ }^{3}$ Department of Medicine, Maastricht University, Maastricht, The Netherlands ${ }^{4}$ Department of Pharmaceutical Gene Modulation, Rijksuniversiteit Groningen, Groningen, Groningen, The Netherlands

${ }^{5}$ Medical Staff, NOC NSF, Arnhem, Gelderland, The Netherlands

\section{Correspondence to} Mr Tom GH Wiggers, Sports Medicine, St Anna Hospital, Geldrop, The Netherlands; t.wiggers@st-anna.nl

Accepted 7 May 2021 Published Online First 26 May 2021

\begin{abstract}
Objective Stem cell therapy is increasingly used for knee osteoarthritis (KOA). We aimed to review the evidence of autologous mesenchymal stem cell therapy on pain, function and severity on imaging in KOA.
\end{abstract}

Design Systematic review of randomised controlled trials (RCTs).

Eligibility criteria RCTs evaluating autologous

mesenchymal stem cell (MSC) therapy on patient-reported outcome measures and disease severity.

Data sources Seven databases were searched until 31

December 2020.

Risk of bias and data synthesis Risk of bias was assessed using the ROB V.2. We used Grading of Recommendations Assessment, Development and Evaluation to appraise the certainty of the evidence. Data were synthesised descriptively.

Results Fourteen RCTs were included. A total of 408 patients with $\mathrm{KOA}$ received MSC therapy derived from bone marrow, adipose tissue or activated peripheral blood. After 1 year, 19 of $26(73 \%)$ clinical outcome measures improved with MSCs compared with control. In the MSC group, patients improved by 1.8-4.4 points on the Visual Analogue Scale (0-10) and 18-32 points of the Knee Osteoarthritis Outcome Score (0-100). Four studies showed better disease severity on imaging after MSC compared with control at 1 year. Ten of $14(71 \%)$ RCTs were at high risk of bias on all outcomes. No serious adverse events were reported after MSC therapy during a maximum of 4 years follow-up.

Conclusion We found a positive effect of autologous MSC therapy compared with control treatments on patientreported outcome measures, and disease severity. The certainty of this evidence was low to very low.

PROSPERO registration number CRD42019120506

\section{INTRODUCTION}

Knee osteoarthritis (KOA) is a chronic progressive disease and a major cause of disability and pain. ${ }^{12}$ The worldwide prevalence proportion of symptomatic KOA aged $\geq 50$ years is $14 \%-38 \%$ (women) and $4 \%-14 \%$ (men), and will continue to rise because of an older and increasingly obese population. ${ }^{1-3}$ Knee joint injuries also increase the likelihood of (early) KOA. ${ }^{4-6}$ Following joint trauma, $20 \%-50 \%$ of people develop osteoarthritis and it is estimated that posttraumatic osteoarthritis is responsible for about $12 \%$ of all osteoarthritis cases. ${ }^{7}$

Current treatment options for KOA are physical activity (exercise), weight loss, intra-articular injections with corticosteroid, hyaluronic acid (HA) or platelet-rich plasma (PRP) and total knee arthroplasty (TKA). ${ }^{3-10}$ Despite their proven efficacy for patients with KOA, not all patients benefit to satisfactory level. For example, exercise is one of the most studied treatment options in KOA and is found effective in reducing pain and improving physical function, but the magnitude of the effects is limited, that is, $12 / 100$ points (95\% CI 10 to 15$)$ and 10/100 points (95\% CI 8 to 13$)$, respectively. ${ }^{11} 12$ A corticosteroid injection also has a limited pain-reducing effect (1 point on a Visual Analogue Scale (VAS), range 0-10) in the short term (up to 3 months), however, this effect is diminished after 6 months. ${ }^{13}$ Intra-articular injection of HA or PRP improves Western Ontario and McMaster Universities Osteoarthritis Index (WOMAC), range 0-96) after 1 year with 14.0 and 29.6 points, respectively. ${ }^{14}$

TKA is the last resort option for individuals that continue to have pain and decreased function despite being treated with conservative treatments. ${ }^{10} 1215$ The overall satisfaction with TKA is high, resulting in improvement of knee symptoms and function after 1 year. ${ }^{16}$ Despite this, TKA leads to reduced function compared with healthy knees, and the intervention is costly. ${ }^{17} 18$ Moreover, after 25 years 18\% needs revision surgery, with overall less favourable outcomes. ${ }^{19}$

Mesenchymal stem cell (MSC) therapy is a treatment option for KOA with high expectations. Stem cells are proposed to have anti-inflammatory and immunomodulatory properties. ${ }^{1520}$ It is hypothesised that stem cells can promote cartilage regeneration and consequently can postpone or avoid the need for TKA. ${ }^{10}$ In our 2017 systematic review on the efficacy of MSC therapy in KOA, we found a positive effect of MSC therapy (2.1-3.4 points improvement on VAS). ${ }^{21}$ However, high methodological heterogeneity across studies and study outcomes being at high risk of bias did not allow for recommending the use of stem cell therapy in clinical practice. ${ }^{21}$ Over the past 3 years, various new randomised controlled trials (RCTs) have become available, making a thoroughly analysis of the available evidence valuable. ${ }^{22-26}$ Our previous review is the only systematic review that focused on autologous stem cell therapy for KOA. Autologous stem cells are better applicable compared with allogeneic stem cells in clinical practice and this restriction makes the interventions more homogenous. Our aim was to assess the efficacy of autologous stem cell therapy compared with any other treatment or placebo in patients with KOA on patient-reported outcome measures (PROMs) and imaging.

\section{MATERIALS AND METHODS}

This systematic review follows the Preferred Reporting Items for Systematic Reviews and MetaAnalyses statement. ${ }^{27}$ 


\section{Research question}

Is intra-articular injection of MSCs in patients with KOA efficacious compared with other treatments, wait-and-see or no treatment on PROMs, pain measures or a validated imaging scoring system?

\section{Primary and secondary outcome measures}

Primary outcome measures: any PROM on knee function, knee pain and knee-related quality of life at 1-year follow-up (eg, WOMAC, VAS score, Knee Osteoarthritis Outcome Score (KOOS) and International Knee Documentation Committee (IKDC) score).

Secondary outcomes: any PROM at any follow-up, other than at 1 year, all radiological and imaging outcomes (eg, the MRI Osteoarthritis Knee Score and the Whole-Organ MRI Score (WORMS)) and incidence of adverse events during follow-up.

\section{Eligibility criteria}

Population: Trials with participants with any degree of primary or secondary osteoarthritis of the knee were included. Trials with participants with one or more focal chondral defects without generalised osteoarthritis were excluded. No age restriction for participants was applied.

Intervention and comparisons: Included trials performed an intra-articular injection of autologous MSCs into the affected knee. All dosages, timing variations and delivery modalities of MSCs were included. Trials including treatment of stem cells combined with another intervention were included. Trials using one knee for intervention and the contralateral knee of the same patient for control were excluded, because PROMs measure outcomes on the patient level; not on the leg-level. Any comparing intervention for KOA (eg, physiotherapy, injections or surgery), placebo or control group (wait-and-see, no treatment) was eligible. Trials using allogeneic stem cells are excluded.

Primary and secondary outcome measures: Trials that assessed the efficacy of intra-articular injection or implantation of stem cells on PROMS, pain or a validated imaging scoring system were included. Another outcome measure was the occurrence of adverse events. Any time point during follow-up was available for inclusion.

Studies: RCTs available in full text were included. Trials using any other study design were excluded.

\section{Search methods}

We used the sensitive search strategy which was developed with help from a research librarian for all databases, by Pas et al. ${ }^{21}$ We systematically searched the literature for trials evaluating the effect of intra-articular stem cell therapy without restrictions of time, language or content. One author (TW) searched the following conventional literature sources for relevant reports of individual studies: PubMed, EMBASE, CINAHL, Web of Science, Cochrane Library, PEDro and SPORTDiscus. Databases were searched until 31 December 2020.

\section{Study selection}

All citations were downloaded into an electronic citation manager (RevMan V.5.3, Copenhagen: The Nordic Cochrane Centre, The Cochrane Collaboration, 2014) by one author (TW), and duplicates were removed. References of included trials were searched for trials that may have been missed during this search. Two authors (TW and MM) screened first titles and then abstracts for eligibility of identified studies. After title and abstract screening, the same two authors independently reviewed full text articles. If full text was not available, the listed contact person of the trials was contacted by e-mail and if necessary, a reminder after 3 weeks was sent. If we received no response, the trial data were considered unavailable. Then, both authors read full-text content and independently assessed eligibility by applying our inclusion criteria. In case of disagreement between reviewers, consensus was sought through discussions or a third reviewer (MW) made the final decision.

\section{Data extraction}

An a priori data extracting sheet was used to extract study characteristics and study outcomes. Data were extracted by one author (TW). Study characteristics included: study design, population, stem cell type, number and timing of injections, delivery method (injection or administered during surgery), concomitant and control intervention and follow-up. Study outcomes included: number of injected cells, outcome measures and adverse events. In case outcome data was not reported, we contacted the trial authors for data availability.

\section{Risk of bias assessment of individual studies}

We used the Risk of Bias V.2 (ROB V.2) tool to assess the risk of bias for each outcome measure per study. ${ }^{28}$ This new tool has a fixed set of items, that is, 'bias arising from the randomisation process', 'bias due to deviations from intended interventions', 'bias due to missing outcome data', 'bias in measurement of the outcome', 'bias in selection of the reported result' and overall risk of bias judgement for each outcome. We assessed risk of bias on the basis of 'adhering to intervention'. Two reviewers (TW and NACB) independently assessed the risk of bias for each outcome within the study, for each follow-up. Each major domain of bias was appraised for every individual outcome measure. We followed the tool's signalling questions and criteria to inform a domain-based appraisal of the risk of bias. The risk of distortion of the outcome estimate was appraised as at 'low', 'some concerns' or 'high' risk of bias. Each outcome measure within a study received an overall risk of bias judgement based on the individual domains; 'low', 'some' or 'high' risk of bias. In case of persistent disagreement between authors, a third reviewer (MW) made the decision.

\section{Data synthesis}

We planned a meta-analysis for those studies that were sufficiently comparable with regards to the intervention, comparison, populations and outcomes. Study characteristics were cross-tabulated and checked for any clinical potential effect modifiers before any analysis was commenced. In case of clinical heterogeneity, or when insufficient data of original studies were available to perform a meta-analysis, a descriptive synthesis was performed.

\section{Certainty of evidence (Grading of Recommendations Assessment, Development and Evaluation)}

For all outcomes, two authors (TW and NACB) independently assessed the certainty of evidence of (possible) benefits of stem cells compared with other treatments using the Grading of Recommendations Assessment, Development and Evaluation (GRADE) approach. ${ }^{29}$ Grading evidence for RCTs starts at 'high quality' and can subsequently be downgraded or upgraded. Factors that could decrease the certainty of evidence were risk of bias, inconsistency, indirectness, imprecision and publication bias for each outcome. Concerning the risk of bias, evidence was downgraded two levels when three or more domains were assessed as high risk of bias. If one or two domains were considered high risk, we downgraded evidence one level. Inconsistency was evaluated by inspecting patient characteristics and outcome 


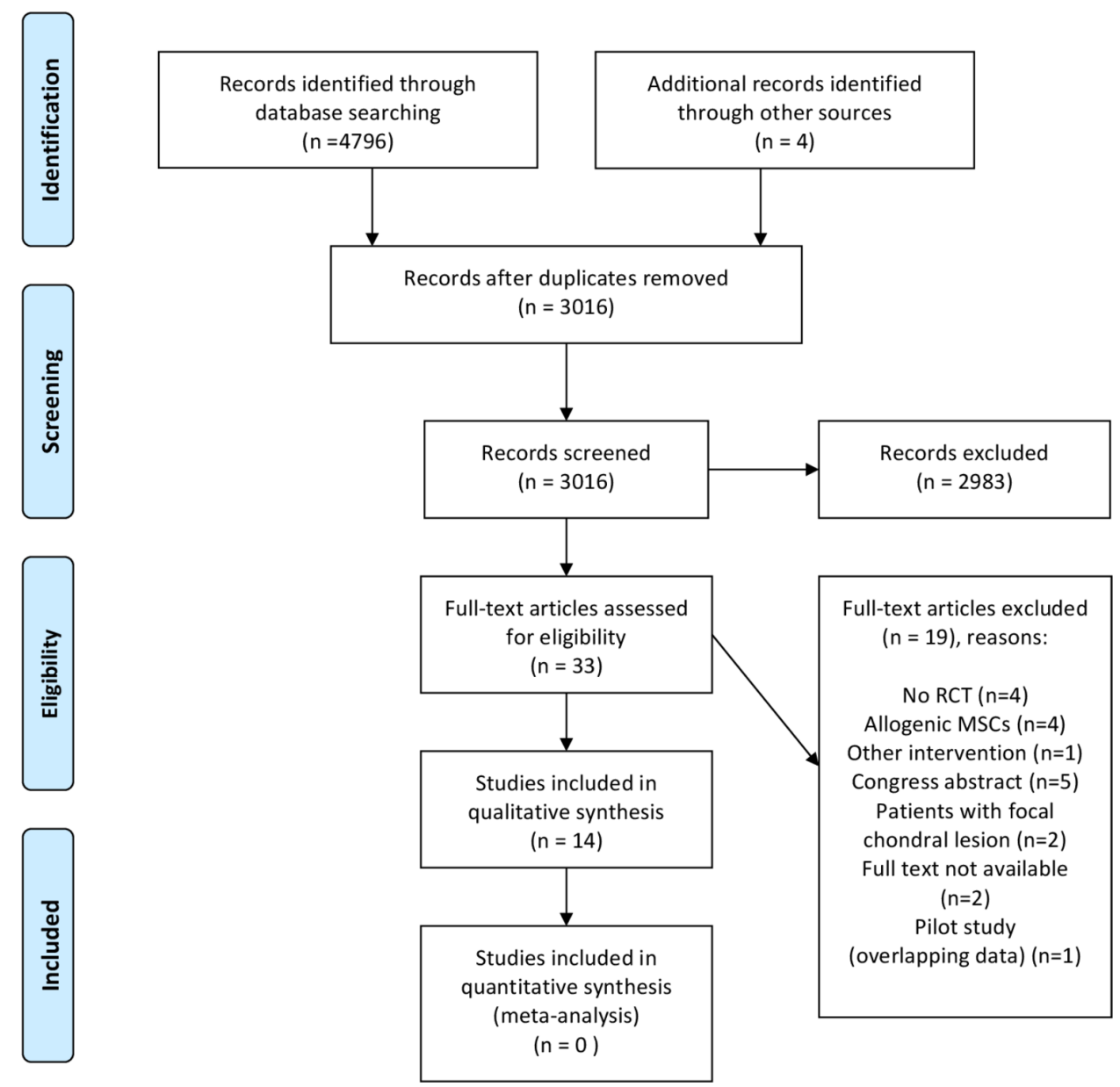

Figure 1 PRISMA flow diagram. Databases searched: PubMed, Embase, Cochrane, Web of Science, CINAHL, Sportdiskus, Pedro. PRISMA, Preferred Reporting Items for Systematic Reviews and Meta-Analyses.

measures. Evidence was downgraded one level if there was a wide variance in estimates across studies, or when there was minimal or no overlap of $95 \%$ CIs, or when studies investigated different study populations. We graded down the evidence by two levels if there was heterogeneity in the patient characteristics and outcomes and if only one study was available for a certain comparison. Evaluation of indirectness was assessed as whether there were differences between trial populations and populations in clinical practice, or when outcomes did not represent relevant outcomes in clinical practice. We graded down the certainty of the evidence by one level if this was the case. Evidence including $<400$ participants was downgraded by one level for imprecision. Evidence could be upgraded in the presence of a large magnitude of effect or a dose-gradient response. The overall certainty of evidence could be rated as 'high', 'moderate', 'low' or 'very low'. The overall certainty of evidence for each outcome was then converted to recommendations for clinical practice ('strength of recommendations').

\section{RESULTS}

\section{Study selection}

The initial search yielded a total of 3016 articles, which were screened on titles. After this, 182 abstracts were assessed of which 36 were selected. We included 14 RCTs. ${ }^{22} 252630-40$ The trial register search yielded no additional articles for inclusion (figure 1). We excluded two abstracts because we were unable to get the full text of 1 abstract and the other one only was available in Chinese. ${ }^{4142}$

\section{Study characteristics}

Online supplemental table 1 summarises the study characteristics. The number of included patients per trial in the intervention group ranged between 10 and 40. A total number of 408 patients were allocated to and treated with a variety of stem cells, and a total of 300 patients were allocated to a control arm. In $11(79 \%)$ trials, Kellgren-Lawrence grade of osteoarthritis was reported and the majority of included patients had grade II (33\%) and grade III (50\%) (3\% had grade I, and 14\% had grade IV). Bone marrow was the most frequently used source of stem cells (8 out of 14 studies; 57\%), adipose tissue was used in 5 trials (36\%) and in one trial (7\%) MSCs from activated peripheral blood were injected. Most trials $(\mathrm{k}=11 ; 79 \%)$ performed 1 MSC injection, ${ }^{22} 25$ 30-32 34-36 38-40 2 (14\%) did 2 MSC injections $^{2637}$ and in 1 trial (7\%) 3 MSC injections were performed. ${ }^{33}$ In four trials (29\%), ${ }^{30} 313339$ MSC injections were used as an additive therapy to surgical interventions, of which arthroscopy and high tibial osteotomy (HTO) in three trials $(21 \%)^{303139}$ and arthroscopic microdrilling in one trial $(7 \%) .{ }^{33} \mathrm{HA}$ as concomitant intervention was used in three trials $(21 \%)^{223033}$ as was PRP injections in three trials $(21 \%) .^{253138}$ The control interventions were HA injection in six trials (43\%), PRP-injection in four trials (29\%), saline-injection in three trials (21\%), dexamethasone 
injection in one trial (7\%) and conservative treatment/exercise in two trials (14\%). At 1 year, 23 clinical outcome measures were available.

\section{Risk of bias assessment}

Figure 2 lists our risk of bias judgements. Thirty-six (84\%) out of 43 clinical outcome measures (13 studies) were assessed as high risk of bias, ${ }^{22} 252630-353^{37-40}$ we had some concerns about bias in 6 clinical outcome measures $\left(14 \% ; 2\right.$ studies) ${ }^{25} 36$ and 1 clinical outcomes measure $\left(2 \% ; 1\right.$ study $^{26}$ was judged to be at low risk of bias. The sources of bias were the randomisation procedure (judged to be as at high risk of bias in 2 studies (14\%), affecting $6(14 \%)$ clinical outcomes), the adherence to intervention (judged to be as at high risk of bias in $5(36 \%)$ studies (affecting 13 (30\%) clinical outcomes), the measurement of the outcomes (judged to be as at high risk of bias in 9 (64\%) studies, affecting 26 (60\%) outcomes), and the risk of bias in selection of the reported results (judged to be as at high risk of bias in 8 (57\%) studies, affecting 24 (56\%) outcomes).

Radiological outcome measures were used in seven trials $(50 \%)$ and reported a total of nine outcomes (six MRI and three X-ray). Six radiological outcome measures in five studies $(67 \%)$ were assessed as high risk of bias, ${ }^{22} 26303138$ we had some concerns about bias in two radiological outcome measures $(22 \%)$ in two studies ${ }^{3637}$ and one radiological outcome measure $(11 \%)$ in one study ${ }^{22}$ was judged to be at low risk of bias. Six trials $(43 \%)$ registered their trial protocol prior to the study's start. $^{22} 2635373840$

\section{GRADE assessment}

Online supplemental table 2 presents the GRADE summary findings for all combinations of MSC therapy and control interventions in the included RCTs. These treatments were evaluated on clinical outcome measures $(14 / 14 ; 100 \%)$, pain score $(10 / 14$; $71 \%)$ and an MRI scoring system (6/14; 43\%). Certainty of evidence for clinical outcome measures was considered low to very low. The evidence was downgraded for risk of bias, inconsistency and imprecisions. We did not assess publication bias due to low study numbers per comparison (ie, $\mathrm{n}<10$ ). The certainty of evidence for MRI outcomes was low to very low (online supplemental table 2). MRI outcome measures were reported in six trials $(43 \%) .^{22} 263036-38$ Several methods for evaluation of cartilage on MRI were used in these 6 RCTs: scoring systems (four studies), assessing cartilage defect size (one study) or cartilage volume (one study). Thus, there was poor generalisability for MRI outcomes. The evidence was downgraded for risk of bias, inconsistency and imprecisions. None of the outcomes were upgraded on the basis of magnitude of effect. Dose-response gradients could not be investigated.

\section{Efficacy of stem cell therapy}

Online supplemental table 3 summaries the outcomes of the intervention groups versus the control groups of all trials. Metaanalysis was precluded because most of the original trial data (ie, central estimates and measures of dispersion for each outcome follow-up) were not available for pooling. Furthermore, studies used different sources of MSCs, different volume (number of cells), and the interventions investigated were heterogenous, that is, in some studies they were combined with surgical procedures. Studies included different comparison groups, and different OA grading further compromising synthesising data in a metaanalysis. Instead, we performed a best evidence synthesis. ${ }^{43}$

\section{Autologous bone marrow-derived stem cells}

The intervention consisted in eight trials (57\%) of MSCs derived from autologous bone marrow. 2225303234353840

\section{Autologous bone marrow-derived stem cells versus HA}

In three trials (27\%), injection of bone marrow-derived stem cells was compared with injection of HA. ${ }^{22} 3032$ Wong et al investigated the effect of bone-marrow derived MSCs injected directly after HTO, arthroscopy and microfracturing in patients with varus knees and osteoarthritis. ${ }^{30}$ The same surgical intervention was performed in control group without harvesting of bone marrow postoperatively and without any injection at that moment. All patients received one injection HA 3 weeks after surgery. After a follow-up of 6, 12 and 24 months both groups achieved improvements on IKDC score. For the interventional group there was a significant additional improvement over the control group after 24 months (IKDC score 7.65 (95\% CI 3.04 to $12.26 ; \mathrm{p}=0.001$ ), where a minimum of 9 points is considered a clinical meaningful improvement. This trial found higher mean Magnetic Resonance Observation of Cartilage Repair Tissue (MOCART) score in the MSC group compared with the control group at 12 months: 62.32 (SD: 17.56) in MSC group and in the control group 43.21 (SD: 13.55) (age-adjusted mean difference of 19.6 ; $95 \%$ CI 10.5 to 28.6 ; $\mathrm{p}<0.001)$. However, no MRI was performed at baseline therefore improvement of cartilage status caused by MSC therapy can not be evaluated in this study.

The study of Lamo-Espinosa et al had two intervention groups (one high-dose and one low-dose bone marrow MSCs, both in addition to one injection HA) and a control group with a HA injection alone. ${ }^{2244}$ Clinical outcomes (VAS and WOMAC) as well as radiological outcome measures (WORMS) were reported at 3 and 6 months and after 1 year. VAS and WOMAC after 4 years were reported separately. ${ }^{44}$ VAS scores did not change in the control group (median VAS from 5 (IQR 3-7) to 4 (IQR 3-5) at 12 months), however, in both intervention groups a significant reduction was found: median VAS from 7 (IQR 5-8) at baseline to 2 (IQR $1-3 ; \mathrm{p}<0.01$ ) at 12 months in low-dose MSC group and median VAS from 6 (IQR 4-8) to 2 (IQR 0-4; $\mathrm{p}<0.01)$ at 12 months in high-dose MSC group. They found a non-significant improvement in WOMAC scores in the control group (29 (IQR 19-38) to 13.5 (IQR 8-33)) and low-dose MSC interventional group (37 (IQR 32-42) to 21.5 (IQR 15-26)) and a significant improvement in the high-dose MSC group (28 (IQR $16-34)$ to 16.5 (IQR 12-19); $\mathrm{p}<0.01$ ). WORMS scores (MRI) after 12 months were not significantly changed in the control and low-dose MSC group and slightly improved in the high-dose MSC group (not statistically significant) compared with baseline. Results after 4 years of follow-up showed an increase of VAS score in the control group (median VAS from 5 (IQR 3-7) at baseline to 7 (IQR 5-7)) and progressive improvement in both intervention groups (median VAS in low-dose MSC group from 7 (IQR 5-8) at baseline to 2 (IQR 2-5; p = 0.01 compared with control group) and high-dose from 6 (IQR 4-8) at baseline to 3 (IQR 3-4, p=0.004 compared with control group)). A significant difference between control group and low-dose MSCs was found for WOMAC scores after 4 years of follow-up $(p=0.01)$, although there was no difference between the control group and the high-dose MSCs group.

Goncars et al investigated the effect of a single injection with autologous bone marrow-derived mononuclear cells versus three injections of HA performed 1 week apart. ${ }^{32}$ After 12 months, KOOS scores improved significantly in both groups and the intervention group performed only better on KOOS pain 


\begin{tabular}{|c|c|c|c|c|c|c|c|}
\hline \multirow{2}{*}{$\begin{array}{l}\text { First author and } \\
\text { year }\end{array}$} & \multirow[t]{2}{*}{ Outcome measure } & \multicolumn{5}{|c|}{ Risk of bias domain } & \multirow{2}{*}{$\begin{array}{l}\text { Overall } \\
\text { risk of } \\
\text { bias }\end{array}$} \\
\hline & & 1 & 2 & 3 & 4 & 5 & \\
\hline \multirow[t]{4}{*}{ Wong $2013^{30}$} & IKDC & $\mathrm{HR}$ & LR & LR & $\mathrm{HR}$ & SC & $\mathrm{HR}$ \\
\hline & Tegner & HR & LR & LR & $H R$ & SC & HR \\
\hline & Lysholm & $\mathrm{HR}$ & LR & LR & $\mathrm{HR}$ & SC & $\mathrm{HR}$ \\
\hline & MOCART & HR & LR & LR & LR & SC & HR \\
\hline \multirow[t]{4}{*}{ Koh $2014^{31}$} & KOOS & $\mathrm{HR}$ & LR & SC & $\mathrm{HR}$ & SC & $\mathrm{HR}$ \\
\hline & VAS & $\mathrm{HR}$ & LR & SC & $\mathrm{HR}$ & SC & $\mathrm{HR}$ \\
\hline & Lysholm & $\mathrm{HR}$ & LR & SC & $\mathrm{HR}$ & SC & $\mathrm{HR}$ \\
\hline & radiology & $\mathrm{HR}$ & LR & SC & $\mathrm{LR}$ & SC & $\mathrm{HR}$ \\
\hline \multirow{5}{*}{$\begin{array}{l}\text { Lamo-Espinosa } 2016 \\
22\end{array}$} & VAS & $\mathrm{LR}$ & LR & $\overline{L R}$ & $H R$ & HR & HR \\
\hline & Knee ROM & LR & LR & LR & $H R$ & HR & HR \\
\hline & WOMAC & LR & LR & LR & $H R$ & $H R$ & HR \\
\hline & $\begin{array}{l}\text { Knee joint space (X- } \\
\text { ray) }\end{array}$ & $\mathrm{LR}$ & LR & LR & $H R$ & LR & HR \\
\hline & WORMS & LR & LR & LR & LR & LR & $L R$ \\
\hline \multirow[t]{2}{*}{ Goncars $2017^{32}$} & KSS & LR & $\mathrm{HR}$ & LR & SC & HR & HR \\
\hline & KOOS & LR & $\mathrm{HR}$ & LR & SC & HR & HR \\
\hline \multirow[t]{2}{*}{ Turajane $2017^{33}$} & Number of TKA & SC & $\mathrm{HR}$ & LR & LR & SC & $\mathrm{HR}$ \\
\hline & WOMAC & SC & $H R$ & LR & HR & HR & HR \\
\hline \multirow[t]{6}{*}{ Emadedin $2018^{34}$} & WOMAC & LR & LR & LR & LR & HR & HR \\
\hline & VAS & LR & LR & LR & LR & $\mathrm{HR}$ & $\mathrm{HR}$ \\
\hline & $\mathrm{MCII}$ & LR & LR & LR & LR & $\mathrm{HR}$ & HR \\
\hline & Walking distance & LR & LR & LR & LR & $H R$ & HR \\
\hline & PASS & LR & LR & LR & LR & $\mathrm{HR}$ & HR \\
\hline & Flexion of knee & LR & LR & LR & LR & $H R$ & HR \\
\hline \multirow{2}{*}{$\begin{array}{l}\text { Lamo-Espinosa } \\
2018^{44}\end{array}$} & VAS & LR & LR & HR & HR & $H R$ & HR \\
\hline & WOMAC & $\mathrm{LR}$ & LR & $H R$ & $H R$ & $H R$ & HR \\
\hline \multirow[t]{5}{*}{ Centeno $2018^{35}$} & VAS & LR & $\mathrm{HR}$ & LR & $\mathrm{HR}$ & $\mathrm{HR}$ & $\mathrm{HR}$ \\
\hline & LEAS & LR & $H R$ & LR & HR & $H R$ & HR \\
\hline & KSS score & LR & $H R$ & LR & HR & $H R$ & HR \\
\hline & SF-12 & LR & $\mathrm{HR}$ & LR & HR & $\mathrm{HR}$ & HR \\
\hline & Knee ROM & LR & $\mathrm{HR}$ & LR & HR & $\mathrm{HR}$ & HR \\
\hline \multirow[t]{5}{*}{ Lee $2018^{36}$} & WOMAC & LR & LR & LR & $\mathrm{LR}$ & SC & SC \\
\hline & VAS & LR & LR & LR & LR & SC & SC \\
\hline & KOOS & LR & LR & LR & LR & SC & SC \\
\hline & Knee ROM & LR & LR & LR & LR & SC & SC \\
\hline & MRI score & $\mathrm{LR}$ & LR & LR & LR & SC & SC \\
\hline \multirow[t]{2}{*}{ Bastos $2020^{25}$} & KOOS & SC & LR & LR & LR & SC & SC \\
\hline & Knee ROM & SC & LR & LR & LR & SC & SC \\
\hline \multirow[t]{4}{*}{ Freitag $2019^{37}$} & NPRS & SC & LR & LR & $\mathrm{HR}$ & LR & $\mathrm{HR}$ \\
\hline & KOOS & SC & LR & LR & HR & LR & HR \\
\hline & WOMAC & SC & LR & LR & HR & $H R$ & HR \\
\hline & MOAKS & SC & LR & LR & LR & SC & SC \\
\hline \multirow[t]{2}{*}{ Lu $2019^{26}$} & WOMAC & LR & LR & LR & LR & LR & $L R$ \\
\hline & VAS & LR & LR & LR & LR & $H R$ & HR \\
\hline
\end{tabular}

Figure 2 Risk of bias analysis. HR, high risk of bias; IKDC, International Knee documentation Committee; KOOS, Knee Osteoarthritis Outcome Score; KSS, Knee Society Score; LEAS, Lower Extremity Activity Score; LR, low risk of bias; MCII, minimum clinically important improvement; MOAKS, MRI Osteoarthritis Knee Scores; NPRS, Numeric Pain Rating Scale; PASS, patient acceptable symptom state; ROM, range of motion; SC, some concerns; SF-36, Short Form Health Survey 36; TKA, total knee arthroplasty; VAS, Visual Analogue Scale; WOMAC, Western Ontario and McMaster Universities Osteoarthritis Index; WORMS, Whole-Organ MRI Score. 
subscale (average improvement of 25.44 points in MSC-group vs 11.37 points (95\% CI not reported) in the control group; $\mathrm{p}<0.05$ ). KSS (Knee Society Score) improved in both groups as well and there was no statistically significant difference between these two groups.

\section{Autologous bone marrow-derived stem cells versus PRP}

In two trials with bone marrow-derived MSCs, the control intervention consisted of PRP. ${ }^{38}{ }^{40}$ In the trial of Lamo-Espinosa et al the intervention group received a single injection MSC+PRP followed by 2 PRP injections after 1 and 2 weeks. ${ }^{38}$ Patients in the control group were treated with three single PRP injections in 3 weeks. After 1 year, there were no statistical significant differences in VAS and WOMAC scores between groups. Radiological outcomes (MRI and X-ray) were unchanged after 1 year in both groups. Anz et al compared a single bone marrow aspirate concentrate injection to a single injection of PRP and found no differences in IKDC and WOMAC scores after 1 year. ${ }^{40}$

\section{Autologous bonemarrow-derived stem cells versus saline}

In the trial of Emadedin et al, 43 patients were injected with MSCs $(n=19)$ or saline $(n=24)$ and followed up for 6 months. ${ }^{34}$ The intervention group improved on WOMAC total score (25.7 points; 95\% CI 16 to 35.4 ) and this was significantly better compared with the control group (WOMAC total 5.5 points; 95\% CI 2.8 to 13.8 ). Painless walking distance was significantly improved in MSC group (+1151 m; 95\% CI 93.4 to $2208.5 \mathrm{~m}$ ). There were no differences between groups on VAS at the end of follow-up (6 months).

\section{Autologous bone marrow-derived stem cells vs. exercise}

In one trial, the control group performed exercise therapy. ${ }^{35}$ In this study, an injection of autologous bone marrow concentrate (BMC) versus exercise therapy in 48 patients was investigated. Because of cross-over of all patients to the interventional group after 3 months, we only included results after 3 months of follow-up. Patients who received BMC injection had significant improvement on the Lower Extremity Activity Scale $(p=0.002)$ and Knee Society Score (KSS) $(\mathrm{p}<0.001)$ compared with control group after 3 months. No differences were found on VAS and knee range of motion (ROM).

\section{Autologous bone marrow-derived MSCs with or without PRP versus corticosteroid injection}

In the trial of Bastos et al, patients were randomised between a single injection with MSC, MSC+PRP or dexamethasone. ${ }^{25}$ After 12 months follow-up, there was a significant improvement of KOOS global for the 2 MSC groups: MD 24.0 (95\% CI 10.3 to 37.7$)$ for MSC group and 22.7 (95\% CI 7.1 to 38.3 ) in the MSC+PRP group (between group difference n.s.). The control group with patients who received dexamethasone injection had a non-significant improvement of KOOS global (MD 17.5 with 95\% CI 3.8 to 31.2 ).

\section{Summary autologous bone marrow-derived stem cells}

At the 1-year follow-up, in total 14 clinical outcome measures were available. Eight (57\%) outcomes were significantly better in comparison with control interventions. ${ }^{22} 253032$ KOOS (scale $0-100)$ was most frequently reported and improved 18-24 points. $^{25} 32$ Strength of evidence was low for six (43\%) clinical outcome measures and very low for eight (57\%) clinical outcomes (online supplemental table 2). There was low to very low strength of evidence for a positive effect of MSC therapy on MRI outcome measures after 1 year. ${ }^{22} 3038$

\section{Autologous adipose-derived MSCs}

The intervention consisted in five trials (36\%) of MSCs derived from autologous adipose tissue. ${ }^{2631363739}$

\section{Autologous adipose-derived MSCs versus PRP}

Koh et al investigated the effect of an injection with adiposederived MSCs plus PRP versus an injection with PRP only. ${ }^{31}$ This injection was given after knee arthroscopy and open-wedge HTO in all patients. After a mean follow-up of 24 months, the MSCPRP group showed significantly greater improvement on KOOS subscale pain $(81.2 \pm 6.9$ MSC-PRP group vs 74.0 $\pm 5.7 \mathrm{PRP}$ only group; $\mathrm{p}<0.001)$ and KOOS subscale symptoms $(82.8 \pm 7.2$ MSC-PRP group vs 75.4 \pm 8.5 PRP only group; $\mathrm{p}=0.006$ ) compared with the control group. VAS score improved in both groups as well, but with a significant greater improvement in the MSC-PRP group $(44.3 \pm 5.7$ at baseline to $10.2 \pm 5.7$ at last follow-up; $\mathrm{p}<0.001)$. Evaluation of the cartilage during secondlook arthroscopy (at time of plate removal) showed better fibrocartilage coverage in the MSC-PRP group compared with the PRP only group: Kanamiya's grade 1: 1 (4.8\%) MSC-PRP vs 11 (47.8\%) in PRP-only group; grade 2: 9 (42.9\%) vs 11 (47.8\%), grade 3 : $8(38.1 \%)$ vs $1(4.3 \%)$ and grade $4: 3(14.3 \%)$ vs none $(0 \%)$.

\section{Autologous adipose-derived MSCs versus saline}

In the RCT of Lee et al, 24 patients with KOA were randomised to MSC therapy or saline injection and followed up for 6 months. ${ }^{36}$ VAS score decreased significantly in the MSC group (from baseline $6.8 \pm 0.6$ to $3.4 \pm 1.5$ at 6 months $(p<0.001)$ ) and did not significantly change in the control group. All KOOS and WOMAC subscales improved significantly where no differences on these scales were found in the control group. Evaluation of cartilage on MRI after 6 months showed no difference in het MSC group and an increase in size of cartilage defect in control group $(\mathrm{p}=0.0051)$.

Autologous adipose-derived MSCs versus conservative treatment In the RCT of Freitag et al 30 patients were randomised to two intervention groups (one or 2 MSC injections) and one control group (conservative treatment (not specified)). ${ }^{37}$ Both intervention groups had a significant decrease in Numeric Pain Rating Scale (NPRS) after 12 months and there was no change in control group $(\mathrm{p}<0.05)$. Between the two treatment groups no significant difference was found (NPRS decreased from 6.7 (SD 1.7) to 2.6 (SD 1.8) and from 6.5 (SD 1.4) to 2.3 (SD 2) in the oneinjection and two-injection group, respectively). Same pattern was found for KOOS subscales pain, symptoms, activities of daily living (ADL), sport and quality of life and WOMAC score: significant improvement in both intervention groups without difference between both groups and no change in control group. MRI after 12 months showed less participants who had progression of cartilage loss in the intervention group compared with the control group (30\% in one-injection group, $11 \%$ in twoinjection group and $67 \%$ in control group; $p=0.043$ ).

\section{Autologous adipose-derived MSCs versus HA}

In the trial of Lu et al patients were randomised to an injection with a product based on human adipose-derived mesenchymal progenitor cells (intervention group) or HA. ${ }^{26}$ Both intervention and control groups had significant improvement 
on WOMAC scores at 12 months follow-up without a difference between both groups $(\mathrm{p}=0.2177)$. WOMAC decreased from $30.83 \pm 19.14$ to $21.70 \pm 17.87(\mathrm{p}=0.0002)$ in the control group and from $34.17 \pm 17.16$ to $27.58 \pm 16.93(\mathrm{p}=0.0001)$ in the intervention group. The Short Form Health Survey score decreased in both groups during 12 months follow-up however the intervention group did significantly better $(\mathrm{p}<0.01)$. MRI results after 12 months favour the intervention group because an increase in volume of cartilage was observed as there was no significant change in the HA group.

\section{Autologous adipose-derived MSCs with or without allogenic cartilage}

In one trial, all patients $(\mathrm{n}=70)$ underwent high tibial osteotomy because of varus KOA. ${ }^{39}$ MSC therapy was performed during this procedure and in half of the patients additionally allogenic cartilage implantation was performed. This resulted in improved Lysholm and KOOS scores in both groups after 1 year (no statistical significance between groups). These scores improved further only in the MSC+ allogenic cartilage group after mean 27 months (improvement of KOOS symptom from baseline mean 24.8 vs $31.6, \mathrm{p}<0.001){ }^{39}$

\section{Summary autologous adipose-derived stem cells}

At the 1-year follow-up, in total eight clinical outcome measures were available and five (63\%) improved significantly more compared with control interventions. ${ }^{26}{ }^{37}$ VAS (scale 0-10) improved 4.1-4.4 points. ${ }^{26}{ }^{37}$ Strength of evidence was low for seven $(88 \%)$ clinical outcome measures and very low for one $(13 \%)$ (online supplemental table 2). There was low to very low strength of evidence for a positive effect of MSC therapy on MRI outcome measures after 1 year. ${ }^{2637}$

\section{Autologous peripheral blood-derived stem cells}

The intervention in one trial (7\%) consisted of MSCs derived from autologous activated peripheral blood. ${ }^{33}$ They compared injections with autologous activated peripheral blood stem cells with or without growth factor addition vs injections with HA. ${ }^{33}$ The primary outcome of this trial was the avoidance of TKA and secondary outcome the WOMAC scores after 1-year follow-up. During this follow-up three patients (15\%) in the control group got a TKA and none of the 40 patients in both intervention groups. All groups improved in WOMAC scores with more improvement in the interventional groups: from 212-218 to 52-75 points in intervention groups versus from 215 to 126 points in the control group $(\mathrm{p}<0.001){ }^{33}$

Summary autologous activated peripheral blood-derived stem cells Only one study (9\%) used activated peripheral blood as source of stem cells. ${ }^{33}$ There was very low strength of evidence for an improvement of WOMAC-score by 137-166 points after MSC therapy (online supplemental table 2). Radiological outcome measures were not available.

\section{Adverse events}

Eleven trials (79\%) reported about adverse events and $3(21 \%)$ did not. Follow-up was 6 months in two trials (14\%), ${ }^{3436} 1$ year in five trials $(36 \%),{ }^{2632} 3337382$ years in three trials $(21 \%)^{303539}$ and 4 years in one trial (7\%). ${ }^{22} 44$ In these 11 trials, 335 patients received MSC therapy and $100(30 \%)$ did report an adverse event. These were all minor adverse events such as temporarily articular pain or mild joint effusion and no serious adverse events. Incidence of adverse events was similar in patients treated with adipose-tissue derived MSCs (47/128 (37\%) in four studies) compared with an intervention using bone marrow-derived MSCs (48/143 (34\%) in six studies). All patients in control groups combined (184 patients), 28 (15\%) adverse events were reported including one $(0.5 \%)$ serious adverse event (knee infection treated with arthroscopic flushing) after HA injection. ${ }^{26}$

\section{DISCUSSION}

We found a positive effect of autologous MSC therapy in KOA on clinical outcome measures $(28 / 43 ; 65 \%)$ and radiological (MRI) outcome measures $(5 / 6 ; 83 \%)$. Clinical outcome measures 1 year after MSC therapy, our primary outcome measure, improved in $19 / 26(73 \%)$ cases. We were not able to pool results in a metaanalysis because of the high heterogeneity between the included trials. Instead, we synthesised data descriptively. Adverse events during the follow-up of the trials were mild and no serious adverse events were reported in patients treated with MSCs. Most outcomes were considered as high risk of bias (84\%), $14 \%$ were considered as some concerns and $2 \%$ as low risk. The strength of evidence for the efficacy for MSCs was low to very low for clinical outcome measures, and was low to very low for radiological outcome measures. Serious adverse events were not reported after MSC therapy during a maximum follow-up of 4 years.

Several possible mechanisms for a positive effect on clinical and radiological outcome measures of MSC therapy are proposed. The first hypothesis is that MSCs have the capacity to differentiate into many different cell types and could be able to regenerate cartilage, however, this is a controversial hypothesis. ${ }^{45}$ The second proposed theory is that MSCs have immunomodulatory and anti-inflammatory effects. ${ }^{10} 15 \quad 2046-48$ This creates an environment which can enhance cartilage healing processes, reduces pain and may result in an improvement of performance perception. ${ }^{15}{ }^{20}$ In this review, cartilage status improved after MSC injection during 1 year of follow-up in 2 of 6 studies (33\%) with MRI outcome measures. ${ }^{26}{ }^{30}$ In three RCTs (50\%), no progression of cartilage loss in the MSC group was found during follow-up in contrast to control groups which had progression of cartilage pathology. ${ }^{22} 3637$ These results could suggest that MSCs have cartilage forming effects and can stop the progression of the disease, however the currently available evidence is insufficient to confirm or reject this hypothesis. The majority of evidence for this hypothesis is from in vitro research, showing MSCs have the ability to reduce inflammation and promote an anti-inflammatory milieu. ${ }^{47}$ The clinical study of Bastos et $a l^{25}$ performed cytokine analysis of synovial fluid. In patients receiving MSCs a significant reduction in interleukin (IL)-10 concentration was found, however, this was also found in the control group that received a corticosteroid injection. No changes in concentrations of IL-17A, interferon-gamma, tumour necrosis factor, IL-2, IL-4 or IL-6 were found in synovial fluid. ${ }^{25}$

We found beneficial results after MSC therapy on patientreported outcomes. In total, we were able to evaluate 408 patients treated with MSC therapy and that is more than twice as many compared with the 2017 review $(n=155) .{ }^{21}$ This shows that the field of MSC therapy for KOA has grown substantially over the past 3-4 years. The number of RCTs in our present study is 14, compared with inclusion of five RCTs and one nonRCT in $2017 .{ }^{21}$ Individual studies are hard to compare directly because of heterogeneity in the characterisation and preparation of the MSCs. This is a consequence of the scientific area of MSC therapy, in which no consensus exists about the ideal source, dose and preparation of stem cells. ${ }^{47}$ There is some evidence 
for a dose-response relation showing more improvement with more injected cells, as shown by the WOMAC scores in the RCT of Lamo-Espinosa et al, and by others (Koh et al Centeno et al). ${ }^{224950}$ In a patient registry of 424 osteoarthritic knee joints treated with BMC a greater pain reduction (adjusted for baseline pain score) was reported for the high-dose group $\left(>4 \times 10^{8}\right.$ nucleated cells) compared with the low-dose group $\left(\leq 4 \times 10^{8}\right.$ nucleated cells). ${ }^{50}$ Despite the positive effects of MSC therapy for KOA, it remains unclear which source, dose and preparation method of MSCs is best. At present, it is unclear if the effects of combined interventions of MSCs with other interventions (ie, biological products (HA or PRP) or surgery) can be attributed to the additional effect of MSCs or an interaction effect of the combined treatments.

We found improvement in knee cartilage status and/or thickness on MRI after MSC therapy. 2226303637 In three of these trials, disease stabilisation was found (compared with progression of disease in the control group), suggesting that MSC therapy can halt the progression of cartilage loss. ${ }^{22} 3637$ Because of the use of several MRI scoring systems, it is difficult to exactly determine the magnitude of increase in cartilage volume in these studies. Ha et al included 17 studies in their review of which 11 studies used an MRI outcome measure. ${ }^{20}$ Nine $(81.8 \%)$ of these outcome measures improved after MSC therapy suggesting a beneficial effect op MSCs on cartilage. In other studies no changes in cartilage status on MRI 6 months after MSC therapy were seen. ${ }^{15174551}$ In the study of Kim et al, there was a significant correlation between improvement in clinical outcomes (IKDC score) and MRI outcome (MOCART). ${ }^{52}$ These findings support the hypothesis MSCs have the capacity to create an environment in which cartilage degeneration stops and possibly can regenerate.

No serious adverse events were reported in patients who received MSC therapy during a maximum of 4 years of follow-up. In 30\% of patients treated with MSC an adverse event occurred. Adverse effects were limited to mild local symptoms as joint pain or mild joint effusion. This is in keeping with the existing literature, where no serious adverse events were reported. ${ }^{10465354}$ Safety in the long-term continues to be a concern as follow-up durations in original studies in our systematic review were only up to 4 years. On the other hand, there is no evidence in the current body of evidence that stem cell therapy in the knee lead to malignancies, a frequently cited potential adverse effect of MSC therapy. ${ }^{556}$ Such major complications of stem cells are only reported in other fields, such as after intravitreal and intrarenal injection. ${ }^{5758}$ In the occurrence of such major complications, factors as comedication, insufficient characterisation of stem cells and by-products in the stem cell injection may have contributed. This emphasises the importance of detailed description of the applied intervention and application of stem cell therapy.

\section{Strengths and limitations}

Strengths of our review include the extensive search strategy in multiple databases, a thorough risk of bias assessment using ROB V.2 and the summary of findings according to the GRADE approach. ${ }^{28} 29$

Our review has a few limitations. First, we were not able to perform a meta-analysis of studies. This is because of the high clinical heterogeneity between the included trials and because insufficient original data could be obtained. This is why we synthesised data descriptively. Second, we included patients with all grades of KOA and therefore we were not able to distinguish the efficacy of MSC therapy in early and advanced stage KOA. Another limitation is that the exact content of the included interventions is heterogeneous, making individual studies hard to compare directly. To overcome some of this heterogeneity, we restricted the inclusion criteria to autologous stem cells and excluded allogeneic stem cell therapy. As far as we know, our present review and previous review ${ }^{21}$ are the only two systematic reviews applying this restriction. The consequence of handling these criteria is that we included fewer RCTs compared with some other systematic reviews and were unable to perform a meta-analysis. We did not apply restrictions to the source of stem cells and included trials using bone marrow-derived, adipose tissue derived and activated peripheral blood derived stem cells, making our results more heterogenous. We could have more optimally evaluate the conduction of stem cell trials by using the 'Minimum Information for Studies Evaluating Biologics in Orthopaedics: PRP and MSCs' consensus checklist by Murray et al. ${ }^{59}$ However, our systematic review shows that there is large heterogeneity in the number of injections, the injected volume (number of cells) and the timing of the injection. It was not possible to investigate if the number, volume and timing of injections affected the outcomes. In one RCT the intervention consisted of both MSC therapy and arthroscopic surgery compared with the control group of three intra-articular injections only. It is unclear if arthroscopic surgery could have distorted the effect of MSC therapy in this study. ${ }^{33}$ In three RCTs both the intervention group and the control group underwent surgical intervention and this can possibly interact with the MSC therapy. ${ }^{303139}$ Lastly, we were not able to evaluate publication bias because of limited number of included RCTs.

\section{Recommendations for research}

We found low quality of evidence for the efficacy of MSC therapy in KOA on clinical and radiological outcome measures. There is high heterogeneity of interventions, control treatments and outcome measures between RCTs. The first step to overcome this heterogeneity is finding the optimal source, dose and frequency of MSC therapy in KOA. Methods of preparation and

\section{What is already known}

- Prevalence of knee osteoarthritis is increasing due to the obesity epidemic and ageing population.

- First line evidence-based treatments like education, exercise therapy and weight reduction do not always yield satisfactory results, and total knee arthroplasty is not always feasible.

- Stem cell therapy is a possible treatment option for those not responding to first line evidence-based treatments, however, the evidence for its efficacy is unknown.

What are the new findings

- We found low to very low quality of evidence for a positive effect of autologous stem cell therapy in knee osteoarthritis on patient-reported outcomes and radiological outcomes.

- There is high heterogeneity in the source, method of preparation and dosage of injected stem cells in included randomised controlled trials (RCTs).

- Serious adverse events seem absent after stem cell therapy; none were reported in RCTs during a maximum follow-up of 4 years. 
characteristics of MSCs used in clinical trials should by reported according to international guidelines. ${ }^{6061}$

Future trials should comply with internationally agreed criteria for the planning, conduct and reporting of clinical trials. ${ }^{59} 62$ Trials need to be registered before trial commencement, and use ROB V.2 to inform the methodological setup of the trials. ${ }^{28}$ Long -term follow-ups (at least 4 years) should be included to monitor unintended effects of the treatment. Commonly used PROMs as VAS, WOMAC and KOOS are preferred in clinical trials. In order to monitoring safety of MSC therapy, accurate registration of adverse events during a long-term follow-up should be applied.

\section{Recommendations for clinical practice}

Key evidence-based treatment options in management of KOA in first line are exercise, weight management and patient education. These non-invasive treatment options are preferred in clinical practice. We found low to very low quality evidence for the efficacy of MSC therapy in KOA. We did not find serious adverse events of MSC therapy. However, follow-up durations in the trials included ranged mostly between 1 and 2 years. One trial had a follow-up duration of 4 years. Therefore, long-term safety of MSC therapy remains unknown. Our findings suggest that MSC therapy could be considered in the treatment of KOA. Given the restricted strength of evidence, application of MSC therapy should be acted on with caution. In our view, MSC therapy should be reserved for those patients with persistent significant pain and disability despite extensive first-line treatments as exercise therapy and weight loss programmes, and when TKA is not feasible. In this patient group, MSC therapy could be considered. Advantages and disadvantages, in light of the limited evidence, should be discussed with the patient in a shared decision process. It should be taken in consideration that stem cell therapy is an expensive therapy and at this moment is not allowed by legal regulations in several countries. Based on our findings, we cannot recommend one source (eg, bone marrow, adipose tissue or peripheral blood) of MSCs over another.

\section{CONCLUSION}

There is low to very low quality evidence for a positive effect of autologous stem cell therapy on clinical outcomes. We found low to very low quality evidence for improved radiology findings after stem cell therapy in KOA. Adverse events of stem cell therapy are limited to mild local symptoms during a maximal follow-up of 4 years (low quality of evidence). Our findings suggest that stem cell therapy could be considered in the treatment of KOA when first line treatments of education, exercise and weight loss has failed, and TKA is not feasible.

Twitter Tom GH Wiggers @wiggersrunning and Marinus Winters @marinuswinters Collaborators not applicable.

Contributors TW contributed to the study conception and design, data collection, and drafting and revision of the manuscript. MW contributed to the study conception and design and drafting and revision of the manuscript. NACB contributed to the data analysis and drafting and revision of the manuscript. $\mathrm{HH}$ contributed to drafting and revision of the manuscript. MM contributed to study conception and design, the data analysis and drafting and revision of the manuscript.

Funding The authors have not declared a specific grant for this research from any funding agency in the public, commercial or not-for-profit sectors.

Competing interests None declared.

Patient consent for publication Not required.

Provenance and peer review Not commissioned; externally peer reviewed.
Supplemental material This content has been supplied by the author(s). It has not been vetted by BMJ Publishing Group Limited (BMJ) and may not have been peer-reviewed. Any opinions or recommendations discussed are solely those of the author(s) and are not endorsed by BMJ. BMJ disclaims all liability and responsibility arising from any reliance placed on the content. Where the content includes any translated material, BMJ does not warrant the accuracy and reliability of the translations (including but not limited to local regulations, clinical guidelines, terminology, drug names and drug dosages), and is not responsible for any error and/or omissions arising from translation and adaptation or otherwise.

\section{ORCID iDs}

Tom GH Wiggers http://orcid.org/0000-0002-8581-8834

Marinus Winters http://orcid.org/0000-0001-5742-7441

Noortje AC Van den Boom http://orcid.org/0000-0003-0652-8546

Maarten H Moen http://orcid.org/0000-0002-3840-7928

\section{REFERENCES}

1 Messier SP, Mihalko SL, Legault C, et al. Effects of intensive diet and exercise on knee joint loads, inflammation, and clinical outcomes among overweight and obese adults with knee osteoarthritis: the idea randomized clinical trial. JAMA 2013;310:1263-73.

2 Bricca A, Juhl CB, Steultjens M, et al. Impact of exercise on articular cartilage in people at risk of, or with established, knee osteoarthritis: a systematic review of randomised controlled trials. Br J Sports Med 2019;53:940-7.

3 Hunter DJ, Bierma-Zeinstra S. Osteoarthritis. Lancet 2019:393:1745-59.

4 Losina E, Weinstein AM, Reichmann WM, et al. Lifetime risk and age at diagnosis of symptomatic knee osteoarthritis in the US. Arthritis Care Res 2013:65:703-11.

5 Muthuri SG, McWilliams DF, Doherty M, et al. History of knee injuries and knee osteoarthritis: a meta-analysis of observational studies. Osteoarthritis Cartilage 2011;19:1286-93.

6 Snoeker B, Turkiewicz A, Magnusson K, et al. Risk of knee osteoarthritis after different types of knee injuries in young adults: a population-based cohort study. Br J Sports Med 2020;54:725-30.

7 Punzi L, Galozzi P, Luisetto R, et al. Post-Traumatic arthritis: overview on pathogenic mechanisms and role of inflammation. RMD Open 2016;2:e000279.

8 Scott CEH, Howie CR, MacDonald D, et al. Predicting dissatisfaction following total knee replacement: a prospective study of 1217 patients. J Bone Joint Surg $\mathrm{Br}$ 2010:92:1253-8

9 Aaboe J, Bliddal H, Messier SP, et al. Effects of an intensive weight loss program on knee joint loading in obese adults with knee osteoarthritis. Osteoarthritis Cartilage 2011;19:822-8.

10 Migliorini F, Rath B, Colarossi G, et al. Improved outcomes after mesenchymal stem cells injections for knee osteoarthritis: results at 12-months follow-up: a systematic review of the literature. Arch Orthop Trauma Surg 2020;140:853-68.

11 Fransen M, McConnell S, Harmer AR, et al. Exercise for osteoarthritis of the knee: a Cochrane systematic review. Br J Sports Med 2015;49:1554-7.

12 Henriksen M, Christensen R, Klokker L, et al. Evaluation of the benefit of corticosteroid injection before exercise therapy in patients with osteoarthritis of the knee: a randomized clinical trial. JAMA Intern Med 2015;175:923-30.

13 Juni P, Hari R, Rutjes AW. Intra-Articular corticosteroid for knee osteoarthritis. Cochrane Database Syst Rev 2015;10:CD005328.

14 Meheux CJ, McCulloch PC, Lintner DM, et al. Efficacy of intra-articular plateletrich plasma injections in knee osteoarthritis: a systematic review. Arthroscopy 2016;32:495-505.

$15 \mathrm{Kim} \mathrm{SH}, \mathrm{Ha}$ C-W, Park Y-B, et al. Intra-Articular injection of mesenchymal stem cells for clinical outcomes and cartilage repair in osteoarthritis of the knee: a meta-analysis of randomized controlled trials. Arch Orthop Trauma Surg 2019:139:971-80.

16 Skou ST, Roos EM, Laursen MB, et al. A randomized, controlled trial of total knee replacement. N Engl J Med 2015;373:1597-606.

17 Shapiro SA, Arthurs JR, Heckman MG, et al. Quantitative T2 MRI mapping and 12-month follow-up in a randomized, blinded, placebo controlled trial of bone marrow aspiration and concentration for osteoarthritis of the knees. Cartilage 2019;10:432-43.

18 Hurley M, Dickson K, Hallett R, et al. Exercise interventions and patient beliefs for people with hip, knee or hip and knee osteoarthritis: a mixed methods review. Cochrane Database Syst Rev 2018:4:CD010842.

19 Evans JT, Walker RW, Evans JP, et al. How long does a knee replacement last? A systematic review and meta-analysis of case series and national registry reports with more than 15 years of follow-up. Lancet 2019;393:655-63.

$20 \mathrm{Ha}$ C-W, Park Y-B, Kim SH, et al. Intra-Articular mesenchymal stem cells in osteoarthritis of the knee: a systematic review of clinical outcomes and evidence of cartilage repair. Arthroscopy 2019;35:277-88.

21 Pas HI, Winters M, Haisma HJ, et al. Stem cell injections in knee osteoarthritis: a systematic review of the literature. Br J Sports Med 2017;51:1125-33.

22 Lamo-Espinosa JM, Mora G, Blanco JF, et al. Intra-Articular injection of two different doses of autologous bone marrow mesenchymal stem cells versus hyaluronic acid in the treatment of knee osteoarthritis: multicenter randomized controlled clinical trial (phase I/II). J Trans/ Med 2016;14:246. 
23 Shapiro SA, Kazmerchak SE, Heckman MG, et al. A prospective, single-blind, placebocontrolled trial of bone marrow aspirate concentrate for knee osteoarthritis. Am J Sports Med 2017;45:82-90.

24 Bastos R, Mathias M, Andrade R, et al. Intra-Articular injections of expanded mesenchymal stem cells with and without addition of platelet-rich plasma are safe and effective for knee osteoarthritis. Knee Surg Sports Traumatol Arthrosc 2018;26:3342-50

25 Bastos R, Mathias M, Andrade R, et al. Intra-Articular injection of culture-expanded mesenchymal stem cells with or without addition of platelet-rich plasma is effective in decreasing pain and symptoms in knee osteoarthritis: a controlled, double-blind clinical trial. Knee Surg Sports Traumatol Arthrosc 2020;28:1989-99.

26 Lu L, Dai C, Zhang Z, et al. Treatment of knee osteoarthritis with intra-articular injection of autologous adipose-derived mesenchymal progenitor cells: a prospective, randomized, double-blind, active-controlled, phase Ilb clinical trial. Stem Cell Res Ther 2019;10:143.

27 Shamseer L, Moher D, Clarke M, et al. Preferred reporting items for systematic review and meta-analysis protocols (PRISMA-P) 2015: elaboration and explanation. BMJ 2015;349:g7647.

28 Sterne JAC, Savović J, Page MJ, et al. Rob 2: a revised tool for assessing risk of bias in randomised trials. BMJ 2019;366:14898

29 Guyatt GH, Oxman AD, Vist GE, et al. Grade: an emerging consensus on rating quality of evidence and strength of recommendations. BMJ 2008;336:924-6.

30 Wong KL, Lee KBL, Tai BC, et al. Injectable cultured bone marrow-derived mesenchymal stem cells in varus knees with cartilage defects undergoing high tibial osteotomy: a prospective, randomized controlled clinical trial with 2 years' follow-up. Arthroscopy 2013;29:2020-8.

31 Koh Y-G, Kwon O-R, Kim Y-S, et al. Comparative outcomes of open-wedge high tibial osteotomy with platelet-rich plasma alone or in combination with mesenchymal stem cell treatment: a prospective study. Arthroscopy 2014;30:1453-60.

32 Goncars V, Jakobsons E, Blums K, et al. The comparison of knee osteoarthritis treatment with single-dose bone marrow-derived mononuclear cells vs. hyaluronic acid injections. Medicina 2017;53:101-8.

33 Turajane T, Chaveewanakorn U, Fongsarun W, et al. Avoidance of total knee arthroplasty in early osteoarthritis of the knee with intra-articular implantation of autologous activated peripheral blood stem cells versus hyaluronic acid: a randomized controlled trial with differential effects of growth factor addition. Stem Cells Int 2017:2017:1-10.

34 Emadedin M, Labibzadeh N, Liastani MG, et al. Intra-Articular implantation of autologous bone marrow-derived mesenchymal stromal cells to treat knee osteoarthritis: a randomized, triple-blind, placebo-controlled phase 1/2 clinical trial. Cytotherapy 2018;20:1238-46.

35 Centeno C, Sheinkop M, Dodson E, et al. A specific protocol of autologous bone marrow concentrate and platelet products versus exercise therapy for symptomatic knee osteoarthritis: a randomized controlled trial with 2 year follow-up. J Trans/ Med 2018; 16:355.

36 Lee W-S, Kim HJ, Kim K-I, et al. Intra-Articular injection of autologous adipose tissuederived mesenchymal stem cells for the treatment of knee osteoarthritis: a phase IIb, randomized, placebo-controlled clinical trial. Stem Cells Trans/ Med 2019;8:504-11.

37 Freitag J, Bates D, Wickham J, et al. Adipose-Derived mesenchymal stem cell therapy in the treatment of knee osteoarthritis: a randomized controlled trial. Regen Med 2019;14:213-30.

38 Lamo-Espinosa JM, Blanco JF, Sánchez M, et al. Phase II multicenter randomized controlled clinical trial on the efficacy of intra-articular injection of autologous bone marrow mesenchymal stem cells with platelet rich plasma for the treatment of knee osteoarthritis. J Trans/ Med 2020;18:356.

39 Kim YS, Chung PK, Suh DS, et al. Implantation of mesenchymal stem cells in combination with allogenic cartilage improves cartilage regeneration and clinica outcomes in patients with concomitant high tibial osteotomy. Knee Surg Sports Traumatol Arthrosc 2020:28:544-54.

40 Anz AW, Hubbard R, Rendos NK, et al. Bone marrow aspirate concentrate is equivalent to platelet-rich plasma for the treatment of knee osteoarthritis at 1 year: a prospective, randomized trial. Orthop J Sports Med 2020;8:232596711990095.
41 Xiaoxia L, Cheng H, Zhi Y. Effectiveness of autologous bone marrow mesenchymal stem cell transplant for knee osteoarthritis. Chinese J Cell Stem Cell 2015:5:96-100.

42 Tan $Y$, Jiang M, Yu H. Therapeutic effect of arthroscopy combined with autologous bone marrow stem cell grafting on knee osteoarthritis. J Tradit Chin Orthop Traumatol 2013:35-8.

43 Higgins JPT, Thomas J, Li T. Cochrane Handbook for systematic reviews of interventions version 6.1 (updated September 2020). Cochrane, 2020. Available: www.training. cochrane.org/handbook

44 Lamo-Espinosa JM, Mora G, Blanco JF, et al. Intra-Articular injection of two different doses of autologous bone marrow mesenchymal stem cells versus hyaluronic acid in the treatment of knee osteoarthritis: long-term follow up of a multicenter randomized controlled clinical trial (phase I/II). J Trans/ Med 2018;16:213.

45 Shin Y-S, Yoon J-R, Kim H-S, et al. Intra-Articular injection of bone marrow-derived mesenchymal stem cells leading to better clinical outcomes without difference in MRI outcomes from baseline in patients with knee osteoarthritis. Knee Surg Relat Res 2018:30:206-14

46 Cui G-H, Wang YY, Li C-J, et al. Efficacy of mesenchymal stem cells in treating patients with osteoarthritis of the knee: a meta-analysis. Exp Ther Med 2016;12:3390-400.

47 Lopa S, Colombini A, Moretti M, et al. Injective mesenchymal stem cell-based treatments for knee osteoarthritis: from mechanisms of action to current clinical evidences. Knee Surg Sports Traumatol Arthrosc 2019;27:2003-20.

48 Musiał-Wysocka A, Kot M, Majka M. The pros and cons of mesenchymal stem cellbased therapies. Cell Transplant 2019;28:801-12.

49 Koh Y-G, Jo S-B, Kwon O-R, et al. Mesenchymal stem cell injections improve symptoms of knee osteoarthritis. Arthroscopy 2013;29:748-55.

50 Centeno CJ, Al-Sayegh $\mathrm{H}$, Bashir J, et al. A dose response analysis of a specific bone marrow concentrate treatment protocol for knee osteoarthritis. BMC Musculoskelet Disord 2015;16:258.

51 Pintat J, Silvestre A, Magalon G, et al. Intra-Articular injection of mesenchymal stem cells and platelet-rich plasma to treat Patellofemoral osteoarthritis: preliminary results of a long-term pilot study. J Vasc Interv Radiol 2017;28:1708-13.

52 Kim YS, Choi YJ, Lee SW, et al. Assessment of clinical and MRI outcomes after mesenchymal stem cell implantation in patients with knee osteoarthritis: a prospective study. Osteoarthritis Cartilage 2016;24:237-45.

53 Koh Y-G, Choi Y-J. Infrapatellar fat pad-derived mesenchymal stem cell therapy for knee osteoarthritis. Knee 2012;19:902-7.

54 Yubo M, Yanyan L, Li L, et al. Clinical efficacy and safety of mesenchymal stem cell transplantation for osteoarthritis treatment: a meta-analysis. PLoS One 2017;12:e0175449

55 Peeters CMM, Leijs MJC, Reijman M, et al. Safety of intra-articular cell-therapy with culture-expanded stem cells in humans: a systematic literature review. Osteoarthritis Cartilage 2013;21:1465-73.

56 Lalu MM, McIntyre L, Pugliese C, et al. Safety of cell therapy with mesenchymal stromal cells (SafeCell): a systematic review and meta-analysis of clinical trials. PLoS One 2012;7:e47559.

57 Thirabanjasak D, Tantiwongse K, Thorner PS. Angiomyeloproliferative lesions following autologous stem cell therapy. J Am Soc Nephrol 2010;21:1218-22.

58 Kuriyan AE, Albini TA, Townsend JH, et al. Vision Loss after Intravitreal Injection of Autologous "Stem Cells" for AMD. N Engl J Med 2017;376:1047-53.

59 Murray IR, Geeslin AG, Goudie EB, et al. Minimum information for studies evaluating biologics in orthopaedics (MIBO): platelet-rich plasma and mesenchymal stem cells. J Bone Joint Surg Am 2017;99:809-19.

60 Dominici M, Le Blanc K, Mueller I, et al. Minimal criteria for defining multipotent mesenchymal stromal cells. The International Society for cellular therapy position statement. Cytotherapy 2006;8:315-7.

61 Mendicino M, Bailey AM, Wonnacott K, et al. MSC-based product characterization for clinical trials: an FDA perspective. Cell Stem Cell 2014;14:141-5.

62 Schulz KF, Altman DG, Moher D, et al. Consort 2010 statement: updated guidelines for reporting parallel group randomised trials. BMJ 2010;340:C332. 